\title{
Density of states of the two-dimensional Hubbard model on a $4 \times 4$ lattice
}

\author{
P. W. Leung \\ Supercomputer Computations Research Institute, Florida State University, Tallahassee, Florida $32306-4052$ \\ Zhiping Liu and Efstratios Manousakis \\ Department of Physics and Center for Materials Research and Technology, \\ and Supercomputer Computations Research Institute, \\ Florida State University, Tallahassee, Florida 32306 \\ M. A. Novotny \\ Supercomputer Computations Research Institute, Florida State University, Tallahassee, Florida 32306-4052
}

Paul E. Oppenheimer

Thinking Machines Corporation, Cambridge, Massachusetts 02142-1264

(Received 21 April 1992)

\begin{abstract}
Using exact diagonalization, we calculate the density of states of the two-dimensional Hubbard model on a $4 \times 4$ square lattice at $U / t=0.5,4$, and 10 , and even number of electrons with filling factors $n$ ranging from a quarter filling up to half filling. We compare the ground-state energy and density of states at $U / t=0.5$ and 4 with second-order perturbation theory in $U / t$ in the paramagnetic phase, and find that while the agreement is reasonable at $U / t=0.5$, it becomes worse as the perturbatively determined (i.e., using Stoner's criterion) boundary of the paramagnetic to spin-density-wave instability is approached. In the strong coupling regime $(U / t=10)$, we find reasonable agreement between the density of states of the Hubbard and the $t-J$ model especially for low doping fractions. In general, we find that at half filling the filled states are separated from the empty states by a gap. At $U / t=10$, the density of states shows two bands clearly separated by a Mott-Hubbard gap of order $U$.
\end{abstract}

\section{INTRODUCTION}

The single-electron density of states in materials such as the oxide superconductors can be probed by photoemission $^{1}$ which can reveal the complexity of the band structure, the validity of a quasiparticle description, and the size of correlation effects. The latter effects can be described in a simple way by Hubbard models where the complexity of the local atomic orbitals is included. In order to make progress in understanding the key features of a material such as the superconducting oxides, it is interesting (if at all possible) to try to understand some of the important electron correlation effects by a simple one-band Hubbard model,

$$
H=-t \sum_{\langle i j\rangle, \sigma}\left(c_{i, \sigma}^{\dagger} c_{j, \sigma}+\text { H.c. }\right)+U \sum_{i} n_{i, \uparrow} n_{i, \downarrow},
$$

where $c_{i, \sigma}^{\dagger}$ creates an electron with spin $\sigma$ at site $i,\langle i j\rangle$ are neighboring sites, and $n_{i, \sigma}=c_{i, \sigma}^{\dagger} c_{i, \sigma}$ is the number operator. Here, we shall study by means of exact diagonalization the spectral function and the density of states of the Hubbard model on a $4 \times 4$ lattice. Unfortunately this system size is too small to make direct comparison with experiments. Thus, it is desirable to be able to carry out similar calculations on larger size lattices. It is, however, very difficult to perform a similar study on larger lattices due to limitations in both computer memory and CPU time. Even though we use translational symmetry to reduce the size of the Hilbert space, this system still has about 10 million states at half filling. There are exact diagonalization studies of the energy eigenvalues at various filling factors for the Hubbard model on the $4 \times 4$ lattice. $^{2}$ The density of states (DOS) calculation, however, is especially demanding since we have to repeat the iterations in our Lanczos ${ }^{3}$ algorithm for each distinct reciprocal lattice vector. Quantum Monte Carlo, which is a possible alternative approach, suffers from well-known problems associated with the fermionic statistics and in addition it is not known how to extract dynamical information directly using Monte Carlo techniques. Nevertheless, the results of such an exact calculation, as the one performed here, are useful for enhancing our understanding of the model and correlation effects and in addition they can be used to infer the validity of other approaches.

In this paper we present results obtained by exact diagonalization for the DOS. We have considered an even number of electrons and filling factors ranging from a quarter filling up to half filling. We have chosen three different values of $U / t$ : a rather small value, $U / t=0.5$, which is expected to be in the weak coupling regime; an intermediate value, $U / t=4$; and a large value, $U / t=10$, which is expected to be in the strong coupling region.

We shall compare our results with simple weak cou- 
pling perturbation theory in the paramagnetic ground state mainly because the superconducting oxides are believed to be in this phase. Within low-order perturbation theory, the system is unstable against a spin-density wave (SDW) at a value of $U=U_{c}(n)$ determined by the Stoner criterion, ${ }^{4}$ which depends on the electron-filling factor $n$. This boundary, within the perturbative approach, is the paramagnetic SDW phase boundary. However, we believe that the actual phase boundary should be significantly different from the results of such a perturbative approach in two dimensions. Since our perturbation theory assumes that the ground state is paramagnetic, we need to know the phase boundary which determines the range of validity of such a perturbation theory. We find that the correlation energy is small even close to the SDW instability and that low-order perturbation theory gives ground-state energy close to the exact value. Hence the ground-state energy alone may not be a sensitive indicator for the validity of the perturbation theory. Weak coupling perturbation theory could be valid provided that either $U / t$ or $n$, or both, are small; namely, even though $U$ may be large, at very low electron filling factors, the electrons do not feel the Coulomb repulsion too often. Although we only perform this calculation on the $4 \times 4$ lattice, the perturbation theory can be easily extended to much bigger lattices once its validity is checked through this comparison with the exact results on small size lattices.

We find that for low enough filling factors, even for values of $U / t$ as large as 10, the density of states of the Hubbard model close to the chemical potential found by exact diagonalization is qualitatively similar to those found at small $U / t$. In addition, even at large $U / t$ and close to half filling, the main peak close to the chemical potential in the spectral function follows the form of the noninteracting dispersion relation with a renormalized bandwidth.

The outline of the paper is as follows. In Secs. II and III we briefly describe how we calculate the DOS using, respectively, exact diagonalization and second-order perturbation theory. In Sec. IV, we discuss and compare the ground-state energies and DOS obtained by the two methods, compare the exact diagonalization results at $U / t=10$ with the results of the $t-J$ model, and discuss the spectral functions of the Hubbard model. Section $V$ contains our conclusions.

\section{DENSITY OF STATES USING THE LANCZOS ALGORITHM}

The spectral function for adding an electron of spin $\sigma$, momentum $\mathbf{k}$, and energy $\omega$ to the ground state is given by

$$
\begin{aligned}
A_{\sigma}^{(+)}(\mathbf{k}, \omega)=\frac{1}{N} \sum_{n} & \quad\left|\left\langle\psi_{n}^{N_{e}+1}\left|c_{\mathbf{k}, \sigma}^{\dagger}\right| \psi_{0}^{N_{e}}\right\rangle\right|^{2} \\
\times & \delta\left(\omega-\left(E_{n}^{N_{e}+1}-E_{0}^{N_{e}}\right)\right),
\end{aligned}
$$

where $E_{n}^{N_{e}}$ and $\psi_{n}^{N_{e}}$ are the eigenenergy and eigenfunction of the system of $N_{e}$ electrons on a lattice with $N$ sites. Similarly, the spectral function for removing an electron from the ground state is given by

$$
\begin{aligned}
A_{\sigma}^{(-)}(\mathbf{k}, \omega)=\frac{1}{N} \sum_{n} & \quad\left|\left\langle\psi_{n}^{N_{e}-1}\left|c_{\mathbf{k}, \sigma}\right| \psi_{0}^{N_{e}}\right\rangle\right|^{2} \\
\times & \delta\left(\omega+\left(E_{n}^{N_{e}-1}-E_{0}^{N_{e}}\right)\right) .
\end{aligned}
$$

The wave function $\psi_{0}^{N_{e}}$ is obtained by the Lanczos algorithm. ${ }^{3}$ The corresponding DOS are defined by

$$
N_{\sigma}^{( \pm)}(\omega)=\sum_{\mathbf{k}} A_{\sigma}^{( \pm)}(\mathbf{k}, \omega)
$$

Because of the symmetry of the up and down spins, we consider adding and removing electrons with up spins only and the spin index $\sigma$ will be omitted.

Consider a particular filling factor where the system has $N_{e}$ electrons and drop the superscript $N_{e}$. From the definition of the one-body Green's function

$$
\begin{aligned}
& G^{(-)}(\mathbf{k}, x)=\left\langle\psi_{0}\left|c_{\mathbf{k}}^{\dagger}(x-H)^{-1} c_{\mathbf{k}}\right| \psi_{0}\right\rangle, \\
& G^{(+)}(\mathbf{k}, x)=\left\langle\psi_{0}\left|c_{\mathbf{k}}(x-H)^{-1} c_{\mathbf{k}}^{\dagger}\right| \psi_{0}\right\rangle .
\end{aligned}
$$

$A^{( \pm)}(\mathbf{k}, \omega)$ can be expressed in terms of $G^{( \pm)}(\mathbf{k}, x)$ by

$$
A^{( \pm)}(\mathbf{k}, \omega)=-\frac{1}{N \pi} \operatorname{Im} G^{( \pm)}\left(\mathbf{k}, E_{0} \pm \omega+i \eta\right)
$$

where $\eta$ is a small positive number. The effect of $\eta$ is to broaden the peaks in the DOS.

$G^{( \pm)}(\mathbf{k}, x)$ have a continued fraction expansion, ${ }^{5}$ for example,

$$
G^{(-)}(\mathbf{k}, x)=\frac{\left\langle\psi_{0}\left|c_{\mathbf{k}}^{\dagger} c_{\mathbf{k}}\right| \psi_{0}\right\rangle}{x-a_{1}-\frac{b_{1}^{2}}{x-a_{2}-\frac{b_{2}^{2}}{x-a_{3}-\cdots}}}
$$

where $a_{i}, b_{i}$ are the coefficients of the tridiagonal matrix generated by the Lanczos iteration using $c_{\mathbf{k}}\left|\psi_{0}\right\rangle / \sqrt{\left\langle\psi_{0}\left|c_{\mathbf{k}}^{\dagger} c_{\mathbf{k}}\right| \psi_{0}\right\rangle}$ as the starting vector. The Lanczos iteration can be stopped when the continued fraction expansion has converged satisfactorily. Details of our implementation of the Lanczos algorithm for this system on the Connection Machine CM-2 will be published elsewhere. $^{6}$

\section{DENSITY OF STATES FROM PERTURBATION THEORY}

To start the perturbation approach, let us transform the Hubbard model to momentum space,

$H=\sum_{\mathbf{k}, \sigma} \epsilon_{\mathbf{k}} c_{\mathbf{k}, \sigma}^{\dagger} c_{\mathbf{k}, \sigma}+\frac{U}{N} \sum_{\mathbf{k}, \mathbf{p}, \mathbf{q}} c_{\mathbf{k}+\mathbf{p}, \uparrow}^{\dagger} c_{\mathbf{q}-\mathbf{p}, \downarrow}^{\dagger} c_{\mathbf{k}, \downarrow} c_{\mathbf{q}, \uparrow}$,

where $\epsilon_{\mathbf{k}}=-2 t\left[\cos \left(k_{x} a\right)+\cos \left(k_{y} a\right)\right]$ is the eigenenergy of the noninteracting ground state. At half filling, because of the nesting of the Fermi surface, the ground state is unstable against spin-density fluctuations for arbitrary $U / t$. Away from half filling, as the hole doping level increases, the critical value of $U / t$ needed for the transition between the paramagnetic phase and the spin- 
density phase also increases. The phase boundary can be found by the Stoner criterion. ${ }^{4}$ The antiferromagneticparamagnetic phase boundary is not known and it is not expected to be reliably determined by such a perturbative approach. For our purposes, however, it is needed in order to know for a given $n$ the critical value of $U=U_{c}(n)$ where perturbation theory which assumes a paramagnetic ground state breaks down. In the following calculations, we consider only perturbation expansions in the paramagnetic phase and we shall compute the DOS within this phase.

We define a time-ordered single-electron Green's function as

$$
G_{\sigma}(\mathbf{k}, t)=-i T\left\langle\psi_{0}\left|c_{\mathbf{k}, \sigma}(t) c_{\mathbf{k}, \sigma}^{\dagger}(0)\right| \psi_{0}\right\rangle,
$$

where $\left|\psi_{0}\right\rangle$ is the ground state of the Hamiltonian given by Eq. (9). It is easy to show that

$$
\frac{1}{N \pi} \sum_{\mathbf{k}} \operatorname{Im} G_{\sigma}(\mathbf{k}, \omega)=-N_{\sigma}^{(+)}(\omega)+N_{\sigma}^{(-)}(\omega),
$$

where $N_{\sigma}^{(+)}(\omega)$ and $N_{\sigma}^{(-)}(\omega)$ are the DOS for adding and removing an electron with spin $\sigma$ defined in Eq. (4).

For different hole doping levels, we calculate the selfenergy up to order $(U / t)^{2}$. The contribution from the term of order $U / t$ just gives the Hartree-Fock result and can be included easily. The second-order term of the electron self-energy $\Sigma(\mathbf{k}, \omega)$ can be written as

$$
\Sigma(\mathbf{k}, \omega)=-i \frac{U^{2}}{N} \sum_{\mathbf{q}} \int \frac{d \nu}{2 \pi} G_{0}(\mathbf{k}-\mathbf{q}, \omega-\nu) \chi_{0}(\mathbf{q}, \nu),
$$

with

$$
G_{0}(\mathbf{k}, \omega)=\frac{1}{\omega-\epsilon_{\mathbf{k}}+i \operatorname{sgn}\left(\epsilon_{\mathbf{k}}-E_{F}\right) \eta},
$$

and

$$
\begin{aligned}
\chi_{0}(\mathbf{q}, \omega)=\frac{1}{N} \sum_{\mathbf{k}}[ & \frac{n_{\mathbf{k}}\left(n_{\mathbf{k}+\mathbf{q}}-1\right)}{\omega+\epsilon_{\mathbf{k}}-\epsilon_{\mathbf{k}+\mathbf{q}}+i \eta} \\
& \left.+\frac{n_{\mathbf{k}+\mathbf{q}}\left(1-n_{\mathbf{k}}\right)}{\omega+\epsilon_{\mathbf{k}}-\epsilon_{\mathbf{k}+\mathbf{q}}-i \eta}\right] .
\end{aligned}
$$

Here $G_{0}$ and $\chi_{0}$ are the Green's function and spin susceptibility, respectively, in the absence of the interaction, $n_{\mathbf{k}}$ is the electron occupation number, and $\eta$ is a broadening parameter used to construct the $\delta$ function.

As mentioned above, we start from a paramagnetic noninteracting state. Consequently, these perturbation calculations are valid only when the actual states are paramagnetic. Figure 1 shows the paramagneticantiferromagnetic phase boundary of the infinite twodimensional lattice found by the Stoner criterion. Only in the neighborhood of $n=1$ in the $(U / t, n)$ phase diagram is the system expected to be antiferromagnetic, which would require a different treatment. ${ }^{7}$ This perturbation expansion is expected to be valid for small values of the product $U n$. Thus it is meaningful to compare the results of our exact diagonalization in the region of the paramagnetic phase where $U n \ll 1$, with results of per-

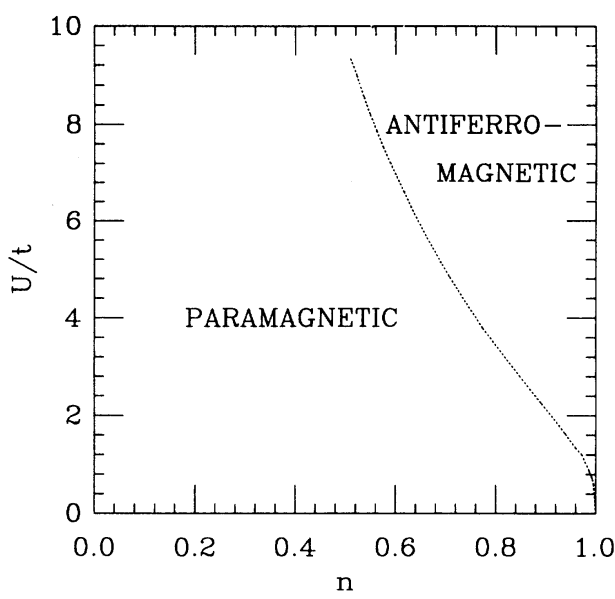

FIG. 1. Phase boundary between the paramagnetic and antiferromagnetic phase of the infinite size Hubbard model. We have not shown the ferromagnetic phase, since it is not relevant to our discussion.

turbation theory. The perturbation treatment can be extended near half filling, where there is antiferromagnetic order in the ground state, using the approach outlined by Schrieffer et al. ${ }^{7}$

\section{RESULTS AND ANALYSIS}

\section{A. Ground-state energy}

Using exact diagonalization, we have calculated the DOS for the parameters $U / t=0.5,4$, and 10 , and filling factors $n=16 / 16,14 / 16,12 / 16,10 / 16$, and $8 / 16$. The ground-state energies for all $N_{e}$ between 16 and 7 are obtained from our Lanczos calculation (see also Table I of the paper by Fano et al. in Ref. 2) and are compared in Table I with the results of perturbation theory.

The ground-state energies can also be calculated using the perturbation theory. On a $4 \times 4$ lattice, except for the cases of 1,2 , and 10 electrons, the unperturbed groundstate energies are highly degenerate. The ground-state

TABLE I. Ground-state energy at various values of $U / t$ and filling factors obtained by exact diagonalization. $N_{e}$ is the number of electrons on a $4 \times 4$ lattice. The numbers in parentheses are calculated from second-order perturbation in $U / t$.

\begin{tabular}{cccr}
\hline \hline$N_{e}$ & $U / t=0.5$ & $U / t=4$ & $U / t=10$ \\
\hline 16 & $-22.34023(-22.34)$ & $-13.62185(-14.2)$ & -7.02900 \\
15 & $-22.49213(-22.48)$ & $-14.66524(-14.5)$ & -8.89301 \\
14 & $-22.64591(-22.65)$ & $-15.74459(-16.2)$ & -10.80701 \\
13 & $-22.79695(-22.80)$ & $-16.72757(-17.1)$ & -12.46957 \\
12 & $-22.94985(-22.95)$ & $-17.72958(-18.1)$ & -14.16438 \\
11 & $-23.10007(-23.10)$ & $-18.64833(-18.9)$ & -15.51372 \\
10 & $-23.25211(-23.25)$ & $-19.58094(-19.9)$ & -16.90356 \\
9 & -21.40396 & -18.55363 & -16.55123 \\
8 & $-19.55701(-19.56)$ & $-17.53490(-17.7)$ & -16.14321 \\
7 & -17.70347 & -16.32054 & -15.34537 \\
\hline \hline
\end{tabular}


energy in a second-order degenerate perturbation calculation involves the diagonalization of the secular matrix in the degenerate Hilbert subspace. ${ }^{8,9}$ This has been calculated in Ref. 8. We use the second-order correlation energy given in Fig. 1 of Ref. 8 to calculate the groundstate energy for $U / t=0.5$ and 4 . The results are also shown in Table I. We observe that the agreement between the perturbation and the exact results is good at all filling factors for $U / t=0.5$, with relative errors of about $0.01 \%$. At $U / t=4$, the larger error (at half filling) is about $4 \%$. Although the errors are within $1 \%$ for smaller filling factors, the DOS in the next section reveal a more subtle disagreement.

\section{B. Density of states}

We now compare the DOS obtained by exact diagonalization and second-order perturbation theory in $U / t$. In the exact diagonalization, we use 100 iterations in the continued fraction expansion, Eq. (8). The parameter $\eta$ in both calculations is taken to be $\eta=0.1 t$. Figures 2 (a) and 2(b) compare the DOS obtained by the two methods. We observe that at $U / t=0.5$ and $n=10 / 16$ [Fig. 2(a)], the agreement is good, as expected. At $U / t=4$ and $n=10 / 16, N^{(-)}$and $N^{(+)}$from perturbation theory
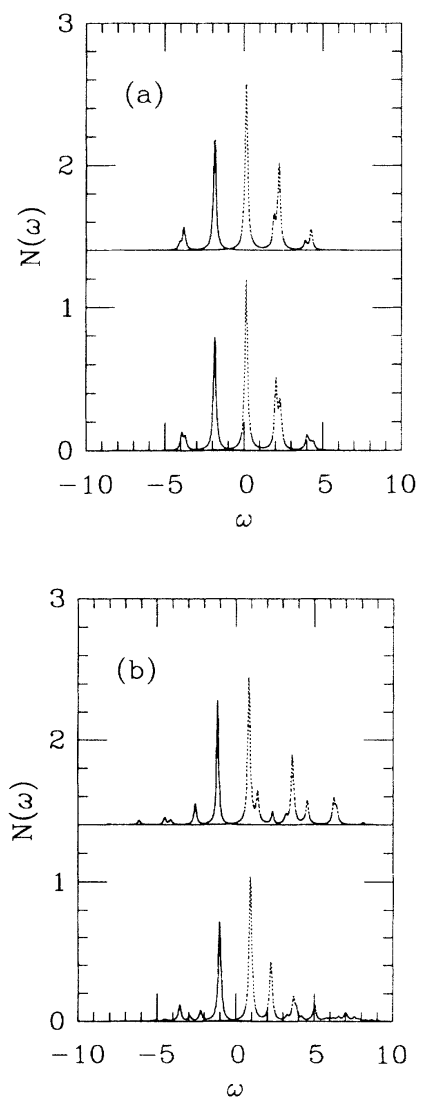

FIG. 2. Comparisons of the DOS obtained by weak coupling perturbation theory (upper graph) and exact diagonalization (lower graph) at $n=10 / 16$ and (a) $U / t=0.5$, (b) $U / t=4$. The solid lines are $N^{(-)}(\omega)$ and the dashed lines are $N^{(+)}(\omega)$ ( $\omega$ is given in units of $t$ ). The perturbation result is shifted upwards for clarity. start to deviate from the exact result. Both calculations agree for both the energy and the strength of the peaks near the chemical potential. The disagreement in both $N^{(-)}$and $N^{(+)}$become worse as one moves away from the chemical potential.

Figures 3, 4, and 5 show the DOS at various $n$ and at $U / t=0.5,4$, and 10 , respectively. Notice that at half filling [(a)] and in all three cases of $U / t$ there is a gap separating the states in $N^{(-)}$from those in $N^{(+)}$. The gap in the case of $U / t=0.5$ is very small and the density of states is close to the noninteracting $(U=0)$ case, and very close to our perturbation theory results. We interpret this gap as the SDW gap (using the weak coupling terminology) while in the strong coupling it becomes the Mott-Hubbard gap. The transition from the strong to weak coupling is believed to be a smooth crossover and the gap in the weak coupling limit is given by $2 \Delta \sim t \exp (-2 \pi \sqrt{t / U})$, while in the strong coupling limit it is of the order of $U$. In the strong coupling perturbation theory in powers of $t / U$ the upper band corresponds to states with one doubly occupied site. As $n$ decreases, more sites are available to accommodate the added electrons without double occupancy, and consequently some of the spectral weight of $N^{(+)}$shifts to the lower band. In our exact diagonalization calculation, we notice that away from half filling there is no gap separating the states contributing to $N^{(-)}$and the states contributing to $N^{(+)}$, i.e., the system becomes metallic. However, in the case of $n=10 / 16$ and for all three values

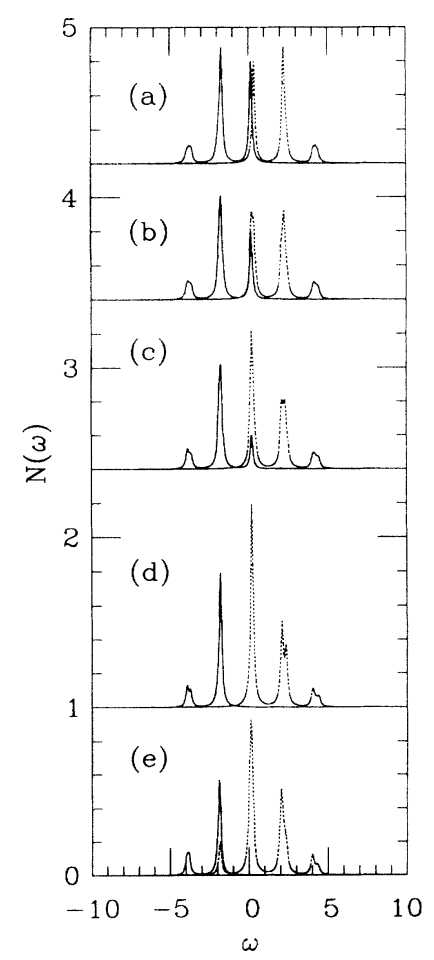

FIG. 3. DOS by exact diagonalization at $U / t=0.5$ and (a) $n=16 / 16$, (b) $n=14 / 16$, (c) $n=12 / 16$, (d) $n=10 / 16$, and (e) $n=8 / 16$. The solid lines are $N^{(-)}(\omega)$ and the dashed lines are $N^{(+)}(\omega)$. All curves except (e) are shifted upwards for clarity. 


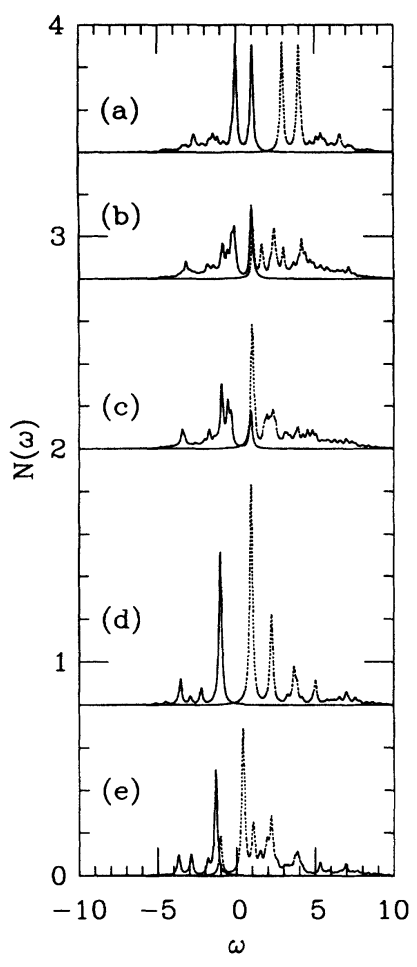

FIG. 4. Same as Fig. 3, except at $U / t=4$.

of $U / t$ we used, there is also a gap separating $N^{(+)}$and $N^{(-)}$. This gap is due to a finite-size effect and can be understood from the noninteracting density of states also: $N^{(+)}(\omega)$ for the three values of $U / t$ considered here and for $n=10 / 16$ share this feature [see (d) of Figs. 3-5]. Ir.

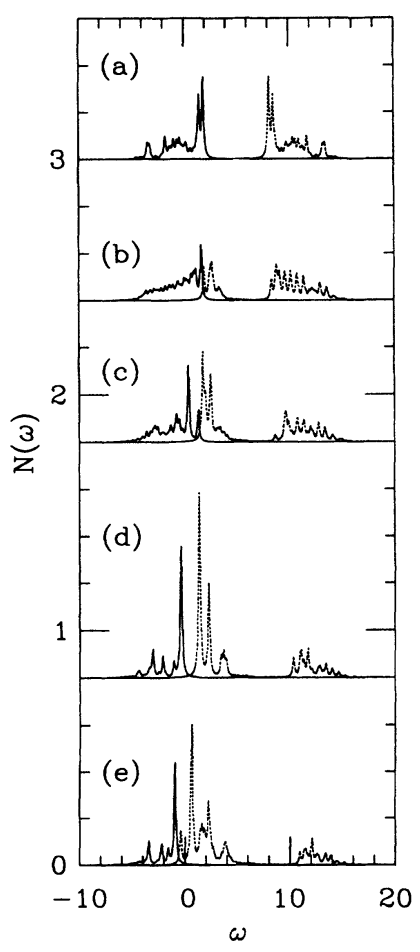

FIG. 5. Same as Fig. 3, except at $U / t=10$. the noninteracting case on a $4 \times 4$ lattice, when we remove six electrons from the half-filled case, the lowest unoccupied state is separated from the highest occupied state by a large discrete amount, and the grouind state of the system is nondegenerate. In the case whare we remove less than six electrons, the noninteracting Fermii lcrel mains unchanged, the system has a degenerate ground state, and the interactions mix these states, create new levels, and move the chemical potential near the highest occupied level of the half-filled ground state. Additional conclusions which can be drawn by studying these results are the following. (a) The structure of the density of states in the neighborhood of the chemical potentials (the lowest energy state in $N^{(+)}$or highest in $N^{(-)}$) is similar for $U / t=4$ and 10. (b) As the density of the electrons is lowered the spectra for various values of $U / t$ become more similar. The latter conclusion was expected, since the effective interaction and the correlations between the electrons are density dependent and in the dilute limit the large $U / t$ is renormalized to a smaller value. We point out that at low filling factors the density of states for all $U / t$ [compare (d) of Figs. 3, 4, and 5] is both qualitatively and quantitatively similar to the noninteracting case (weak coupling limit, i.e., Fig. 3).

At $U / t=10$ there is a clear pseudogap for all values of filling factors as shown in Fig. 5. However, the chemical potential falls well below the gap for the doping levels away from the half filling. Thus in our calculation the Hubbard model behaves as a Mott insulator only at half filling, while below half filling it always acts as a metal.

\section{Comparison with $t-J$ model}

We now compare the Hubbard model at large $U / t$ with the $t-J$ model on the same $4 \times 4$ lattice. Using the relation between the parameters of the two models, $J=4 t^{2} / U$, the largest value in our calculation $U / t=10$ corresponds to $J / t=0.4$ in the $t-J$ model. Since the $t-J$ model is restricted in the single-occupancy subspace its DOS has only the lower Hubbard band. To make a comparison between the DOS of the two models, we truncate the upper band and rescale the lower band of $N^{(+)}$of the Hubbard model so that the areas under $N^{(+)}$are the same as that of the $t-J$ model. The results are shown in Figs. 6(a) and 6(b). The density of states of the $t$ $J$ model are taken from Ref. 12. We find that the $t-J$ model can produce the essential features of the DOS of the Hubbard model at this $U / t$ value at the lower filling factor. Namely, the best agreement is found at lower filling factors $(n=8 / 16)$ where the chance of having doubly occupied sites in the Hubbard model is smaller.

\section{Spectral functions}

In Figs. 7, 8, and 9 we plot the spectral functions at all distinct reciprocal lattice vectors $\mathbf{k}$ at $U / t=10$ and $n=16 / 16,14 / 16$, and $10 / 16$, respectively. Figure 7 is the half-filled case and we notice that the system is a Mott insulator with the chemical potential in the middle of a gap well separating the occupied from the un- 
occupied states. The main peak of $A^{(+)}(\mathbf{k}, \omega)$ for given $\mathbf{k}$ is at an energy $\omega=\omega(\mathbf{k})$ and if we order the various $\mathbf{k}$ values according to the order of increasing energy, they follow the same order as the dispersion relation $\omega(\mathbf{k})=\sqrt{\Delta^{2}+4 t^{2}\left(\cos k_{x}+\cos k_{y}\right)^{2}}$ which can be found by a SDW calculation. ${ }^{7}$

Below half filling (Figs. 8 and 9) we can also find prominent quasiparticle peaks in $A^{( \pm)}(\mathbf{k}, \omega)$ near the value of the chemical potential (which is shown by a vertical line in these figures). In Fig. $8(n=14 / 16)$ the quasiparticle peaks for $\mathbf{k}=(0,0)$ and $\left(0, \frac{\pi}{2}\right)$ are in the $A^{(-)}$spectrum. Note that in the noninteracting case $(U=0)$ with $n=14 / 16$, the states with $\mathbf{k}=(0,0)$ or $\left(0, \frac{\pi}{2}\right)$ lie below the Fermi surface and thus $A^{(-)}$has the peak below the Fermi energy while $A^{(+)}$is zero. Similarly the quasiparticle peaks at $\mathbf{k}=\left(\frac{\pi}{2}, \pi\right)$ and $(\pi, \pi)$ are in the $A^{(+)}$spectrum as in both at $U=0$ and at $U / t=10$. However, for the states $\mathbf{k}=(0, \pi)$ and $\left(\frac{\pi}{2}, \frac{\pi}{2}\right)$ the quasiparticle peaks are on the $A^{+}$and $A^{-}$spectra, respectively, although in the noninteracting case both of these states are on the Fermi surface. This can be understood by means of a simple perturbation calculation. Because of the degeneracy, the single particle states with these two momenta can
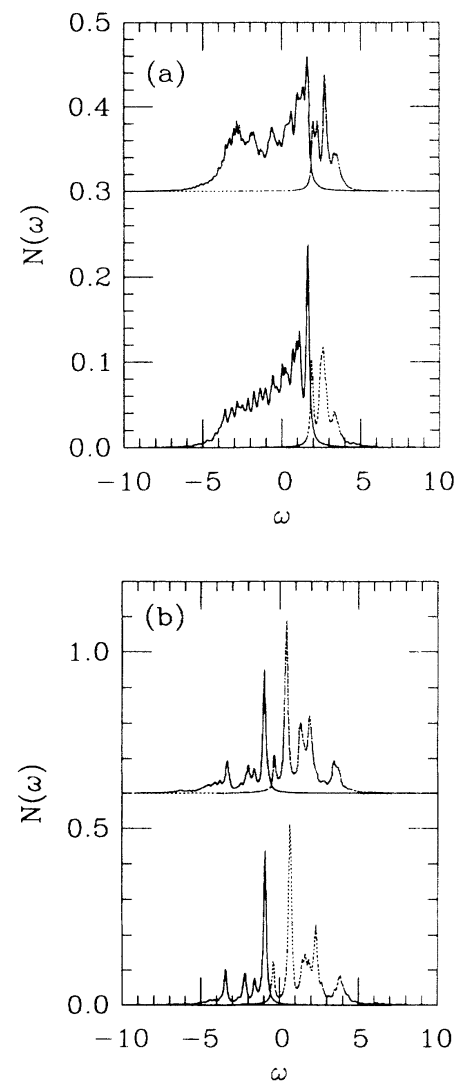

FIG. 6. Densities of states of the Hubbard (lower graphs) and $t-J$ (upper graphs) models at (a) $n=14 / 16$, and (b) $n=8 / 16$. The solid lines are $N^{(-)}(\omega)$ and the dashed lines are $N^{(+)}(\omega)$. The upper bands in the Hubbard model are truncated and the lower bands of $N^{(+)}$are rescaled (see text). Densities of states of the $t-J$ model are from Ref. 12 and are shifted by an arbitrary amount in $\omega$ to facilitate comparisons.

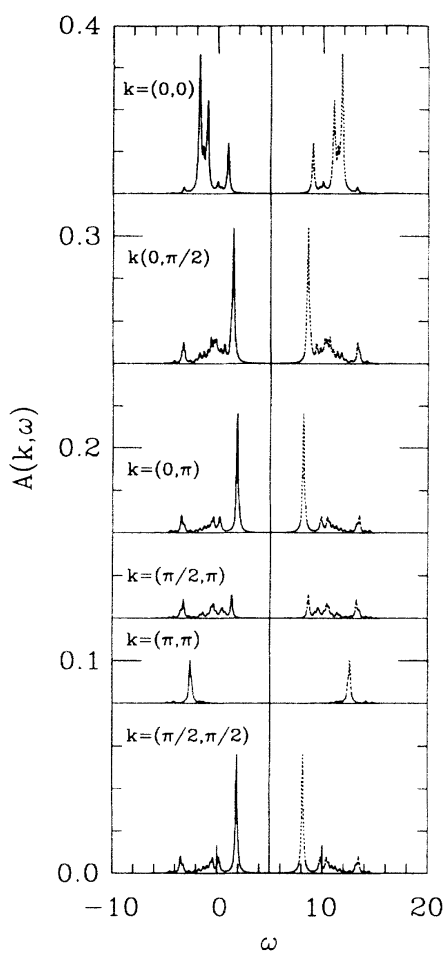

FIG. 7. Spectral functions at $U / t=10$ and $n=16 / 16$. The vertical line is the chemical potential $\mu=5.0 t$.

be occupied, empty or partially occupied for $n=14 / 16$, depending on the choice of the noninteracting ground state; namely, there are 29 degenerate ground states in the Hilbert space of zero total spin and zero momentum. We restrict ourselves in the zero momentum Hilbert sub-

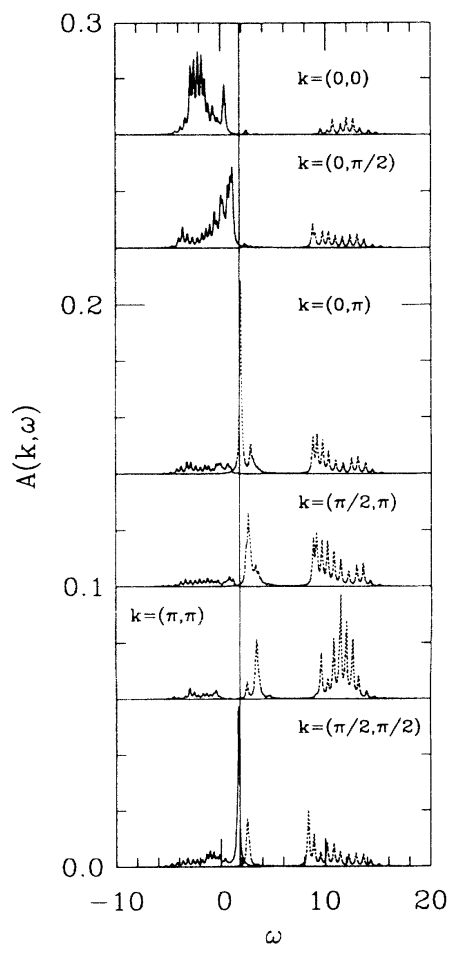

FIG. 8. Spectral functions at $U / t=10$ and $n=14 / 16$. The vertical line is the chemical potential $\mu=1.788 t$. 


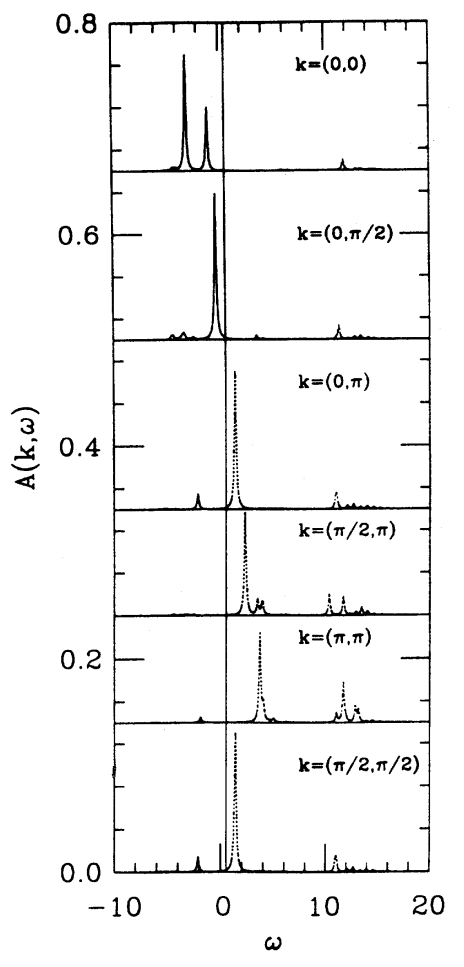

FIG. 9. Spectral functions at $U / t=10$ and $n=10 / 16$. The vertical line is the chemical potential $\mu=0.519 t$.

space because our exact diagonalization is also performed in this subspace. Using first-order degenerate perturbation theory, one can readily show that once the Hubbard interaction is turned on, there is a singly degenerate lowest energy state with zero momentum (i.e., a linear combination of eight noninteracting states with $d$-wave symmetry) in which the single particle states with momenta $\left( \pm \frac{\pi}{2}, \pm \frac{\pi}{2}\right)$ are occupied, while the states with $\mathbf{k}=(0, \pi)$ and its rotations by $\frac{\pi}{2}$ are empty. This perturbation result clearly explains the exact diagonalization result, as is seen in Fig. 8. The spectral functions for $n=10 / 16$ are shown in Fig. 9. There are quasiparticle states for

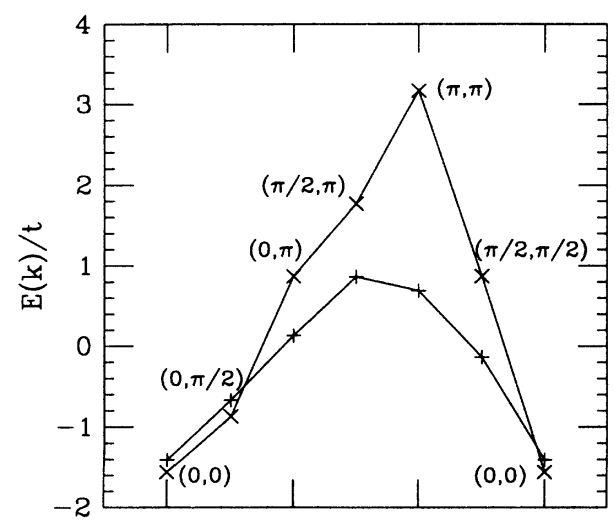

$\mathbf{k}$

FIG. 10. Energies of the quasiparticle states relative to the chemical potential at $U / t=10$ and $n=10 / 16(\times), n=$ $14 / 16(+)$. $\mathbf{k}=(0,0)$ and $\left(0, \frac{\pi}{2}\right)$ in the $A^{(-)}$spectrum while for all other momenta the quasiparticle peaks are in the $A^{(+)}$. This is in qualitative agreement with the noninteracting case, where for $n=10$ the states with $\mathbf{k}=(0,0)$ and the four states obtained from $\left(0, \frac{\pi}{2}\right)$ by $\frac{\pi}{2}$ rotations are completely filled, while the rest are empty.

Figure 10 shows the dispersion relations of the quasiparticle states for both cases $(n=8 / 16$ and $14 / 16)$. The quasiparticle peaks in the spectral functions follow the minima $[$ at $\mathbf{k}=(0,0)]$ and the maxima $[$ at $\mathbf{k}=(\pi, \pi)$ ] of a noninteracting dispersion curve with a renormalized bandwidth. The bandwidth $W$ of the quasiparticle band depends strongly on doping, namely, $W / W_{0} \simeq 0.59$ for $n=10 / 16$ and $W / W_{0} \simeq 0.28$ for $n=14 / 16$, where $W_{0}$ denotes the noninteracting bandwidth $\left(W_{0}=8 t\right)$. Qualitatively similar results are also obtained for the other filling factors.

\section{SUMMARY}

We have presented the ground-state energies and DOS of the Hubbard model on a $4 \times 4$ lattice at various filling factors and $U / t=0.5,4$, and 10 using exact diagonalization. We have also compared them with results for the DOS and the ground-state energies obtained by secondorder perturbation in $U / t$. We find agreement with the results of perturbation theory for small $U / t(U / t=0.5)$ and the agreement starts to become worse at $U / t=4$. At large values of $U / t$ and low filling factors we find some qualitative agreement with perturbation theory. The latter conclusion was expected since the effective interaction and the correlations between the electrons are density dependent and in the dilute limit, the large $U / t$ is renormalized to a smaller value. We also find that the structure of the density of states in the neighborhood of the chemical potential (i.e., the lowest energy state in $N^{(+)}$or the highest in $\left.N^{(-)}\right)$is similar for $U / t=4$ and 10. At large $U / t(U / t=10)$ there is clearly a pseudogap for all values of the filling factors as shown in Fig. 5. However, the chemical potential falls well below the gap except in the case of half filling where it lies in the middle of the gap. Thus in our calculation the Hubbard model behaves as a Mott insulator only at half filling, while below half filling it acts as a metal.

We have also compared our results for the DOS at $U / t=10$ with the DOS of the $t-J$ model at the corresponding $J / t$. We find that the $t-J$ model can reproduce the essential features of the DOS of the Hubbard model (Fig. 6) at low filling factors where the chance of double occupancy is low. Finally, we find that the spectral functions are well described by a quasiparticle picture even at large $U / t$, with a quasiparticle bandwidth which increases upon doping.

We shall avoid direct comparison with the experiments as there is fundamental disagreement between the results found in exact diagonalization studies of the DOS and the experimental photoemission and inverse photoemission results as discussed in Ref. 10.

After this work was completed, we learned that Dagotto, Ortolani, and Scalapino, ${ }^{10}$ and Feng and 
White ${ }^{11}$ have independently calculated the DOS of the Hubbard model on a $4 \times 4$ lattice using exact diagonalization.

\section{ACKNOWLEDGMENTS}

We wish to thank E. Dagotto, J. R. Schrieffer, and P. Schlottmann for useful discussions, and J. Galán for useful communication. This work was supported by the Florida State University Supercomputer Computations Research Institute (SCRI) which is partially funded by the U.S. Department of Energy through Contract No. DE-FC05-85ER25000. The computations were performed on the Thinking Machines Corporation CM-2 Connection Machine at SCRI.
${ }^{1}$ J. W. Allen et al., Phys. Rev. Lett. 64, 595 (1990); T. Takahashi et al., Physica C 170, 416 (1990); H. Matsuyama et al., ibid. 160, 567 (1989).

${ }^{2}$ G. Fano, F. Ortolani, and A. Parola, Phys. Rev. B 42, 6877 (1990); A. Parola et al., ibid. 43, 6190 (1991); A. Moreo, ibid. 45, 4907 (1992).

${ }^{3}$ J. K. Cullum and R. A. Willoughby, Lanczos Algorithms for Large Symmetric Eigenvalue Computations (Birkhauser, Boston, 1985).

${ }^{4}$ D. Penn, Phys. Rev. 142, 350 (1966).

${ }^{5}$ V. Heine, in Solid State Physics, edited by H. Ehrenreich, F. Seitz, and D. Turnbull (Academic, New York, 1980), Vol.
35.

${ }^{6} \mathrm{P}$. W. Leung and P. E. Oppenheimer (unpublished).

${ }^{7}$ J. R. Schrieffer, X. G. Wen, and S. C. Zhang, Phys. Rev. B 39, 11663 (1989).

${ }^{8}$ J. Galán and J. A. Vergés, Phys. Rev. B 44, 10093 (1991). ${ }^{9}$ B. Friedman, Europhys. Lett. 14, 495 (1991).

${ }^{10}$ E. Dagotto, F. Ortolani, and D. Scalapino, Phys. Rev. B 46, 3183 (1992).

${ }^{11} \mathrm{G}$. Feng and S. R. White (unpublished).

${ }^{12}$ E. Dagotto, A. Moreo, F. Ortolani, D. Poilblanc, J. Rierra, and D. Scalapino, Phys. Rev. B (to be published). 